\title{
Clinical characteristics and prognostic analysis of Krukenberg tumor
}

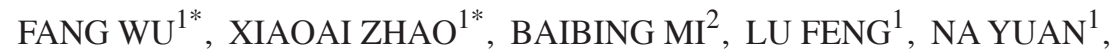 \\ FUXI LEI ${ }^{1}$, MIN LI $^{1}$ and XINHAN ZHAO ${ }^{1}$ \\ ${ }^{1}$ Department of Medical Oncology, The First Affiliated Hospital of Xi'an Jiaotong University School of Medicine; \\ ${ }^{2}$ Department of Epidemiology and Biostatistics, School of Public Health, Xi'an Jiaotong University \\ Health Science Center, Xi'an, Shaanxi 710061, P.R. China
}

Received May 18, 2015; Accepted August 14, 2015

DOI: $10.3892 / \operatorname{mco} .2015 .634$

\begin{abstract}
Krukenberg tumor is a rare metastastic tumor of the ovary, characterized by poor prognosis. In order to analyze the clinical characteristics and prognostic factors, we retrospectively investigated 128 patients who were diagnosed with Krukenberg tumor between January, 1990 and December, 2010. The median patient age was 48 years. The median overall survival (OS) of Krukenberg tumor for all patients was 16 months (95\% CI: 15-19 months). The median OS among patients with Krukenberg tumors of gastric, colorectal, breast and other origins (including appendix, gallbladder, small intestine and unknown primary) was 11 , 21.5, 31 and 19.5 months, respectively $(\mathrm{P}<0.0001)$. In the univariate analysis, synchronous metastasis, no chemotherapy, ovarian metastasis beyond the pelvis, ascites and no metastasectomy were identified as significant poor prognostic factors. The multivariate analysis suggested that synchronous metastasis $(\mathrm{P}=0.0080)$, pelvic invasion $(\mathrm{P}=0.0138)$, ascites $(\mathrm{P}<0.0001)$ and no metastasectomy $(\mathrm{P}=0.0060)$ were independent factors for predicting unfavorable OS. It was suggested that the prognosis of Krukenberg tumor is dismal and ovarian metastasectomy may prove beneficial. Adequate treatment planning is required for this group of patients.
\end{abstract}

\section{Introduction}

Krukenberg tumor is a rare metastatic signet ring cell tumor of the ovary, accounting for $1-2 \%$ of all ovarian tumors. The stomach is the primary site in the majority of Krukenberg

Correspondence to: Professor Xinhan Zhao, Department of Medical Oncology, The First Affiliated Hospital of Xi'an Jiaotong University School of Medicine, 227 Yanta West Road, Xi'an, Shaanxi 710061, P.R. China

E-mail: zhaoxinhanprof@163.com

*Contributed equally

Key words: Krukenberg tumor, ovarian metastasis, clinical characteristic, overall survival, prognostic factor tumor cases, followed by carcinomas of the colon, appendix and breast, particularly invasive lobular carcinoma (1). The eponym was attributed to this tumor following the description of 5 cases by Friedrich Krukenberg (1871-1946) in 1896, who described it as being common among young women, presenting with ascites, an uneven knobby ovarian surface and lymphatic involvement (2). These tumors are characterised by uncertain pathogenesis, challenging etiological diagnosis and poorer prognosis compared with their primaries. Previously, any metastatic ovarian cancer was referred to as Krukenberg tumor; however, Novak and Gray (3) created new diagnostic criteria to eliminate any confusion. Accordingly, a mucin-secreting signet ring cell carcinoma in the dense fibroblastic stroma of the ovary is referred to as Krukenberg tumor. The diagnosis of Krukenberg tumor is currently based on the diagnostic criteria of the World Health Organization based on the pathological description by Serov and Scully (4). The presence of the following characteristics is required for diagnosis: Stromal involvement, mucin-producing neoplastic signet ring cells and ovarian stromal sarcomatoid proliferation.

Krukenberg tumor is considered as a late-stage disease with poor prognosis and may account for 30-40\% of metastatic cancers to the ovaries (5). The treatment approach to these metastatic ovarian tumors remains controversial. To date, treatment mainly consists of ovarian metastasectomy, chemotherapy or radiotherapy; however, the optimal treatment has not yet been established. As the biological behavior and clinical outcome of Krukenberg tumors are rarely summarized, the aim of this retrospective study was to analyze the characteristics and outcome of all patients with Krukenberg tumors over a 20-year period, evaluate the clinical characteristics of such tumors and investigate the prognostic factors.

\section{Patients and methods}

Patient characteristics. Patients who were diagnosed with Krukenberg tumor between January, 1990 and December, 2010, were retrospectively identified from the database of the Department of Oncology, the First Affiliated Hospital of Xi'an Jiaotong University School of Medicine, Tumor Hospital of Shaanxi and the Department of Oncology, People's Hospital of Shaanxi. The data were obtained from the patients' medical 
records and pathology reports. Clinical and pathological variables included age, menopausal status, size of ovarian metastasis of pathologic gross specimen, pathology reports, primary tumor site and subsequent therapy. The quality of the cancer registry database was reviewed and approved by the Ethics Committee of the First Affiliated Hospital of Xi'an Jiaotong University School of Medicine. Overall survival (OS) was calculated from the date of diagnosis of the primary tumor or ovarian metastasis to the date of death or last follow-up. If the time interval between the diagnosis of the primary tumor and that of the ovarian metastasis exceeded 6 months, the metastasis was defined as metachronous in the present study.

Statistical analysis. Statistical analysis was performed using SPSS software, version 17.0 (SPSS Inc., Chicago, IL, USA). A two-sided $\mathrm{P}<0.05$ was considered to indicate a statistically significant difference. The Kaplan-Meier method was used to calculate survival. Clinically relevant variables were analyzed univariately for their association with OS using the log-rank test. The independent prognostic significance of variables in terms of survival was determined in a multivariate analysis using the Cox proportional hazards regression model and the estimates are presented as hazard ratio (HR) with $95 \%$ confidence interval (CI).

\section{Results}

Clinical characteristics. A total of 128 patients were enrolled in this study. The baseline patient characteristics are listed in Table I. The median age at diagnosis of Krukenberg tumor was 48 years (range, 27-65 years). The most common primary tumors were located in the colorectum $(58,45.31 \%)$ and the stomach $(41,32.03 \%)$. Krukenberg tumors were more common in premenopausal women $(75.78 \%)$ rather than in postmenopausal women. Of the 128 patients, $92(71.87 \%)$ had metachronous ovarian metastasis. The treatment options for Krukenberg tumor include metastasectomy and chemotherapy: Metastasectomy was performed in 114 patients (89.06\%), whereas 14 patients (10.94\%) did not undergo surgery due to additional metastatic lesions; chemotherapy was administered to 89 patients $(69.53 \%)$. The surgical procedures included unilateral or bilateral adnexectomy and hysterectomy with bilateral adnexectomy. The main chemotherapeutic drugs included cisplatin, carboplatin, oxaliplatin, docetaxel and 5-fluorouracil. The majority of the patients received 2- or 3 -drug combinations, usually for 4-6 cycles. The majority of the cases $(98,76.56 \%)$ exhibited bilateral ovarian involvement. The median ovarian tumor size was $9.6 \mathrm{~cm}$ (range, $4-18 \mathrm{~cm}$ ), with $58.60 \%$ of the patients having tumors sized $\geq 10 \mathrm{~cm}$. A total of 63 patients $(49.22 \%)$ presented with ascites, while 65 patients $(50.78 \%)$ had no apparent ascites at diagnosis. A total of 71 patients $(55.47 \%)$ had ovarian metastasis, while 57 patients (45.53\%) had combined metastases outside the ovaries, including the pelvis, bone, lung and other distant organs.

Prognosis of Krukenberg tumor. The OS of the 128 patients ranged between 5 and 52 months. The median OS was 16 months (95\% CI: 15-19 months) (Fig. 1). Among all patients, the mean OS for tumors originating in the breast
Table I. Baseline patient characteristics $(n=128)$.

Characteristics

Patients, no. (\%)

$\begin{array}{lc}\text { Age, years } & \\ \text { Median (range) } & 48(27-65) \\ \text { Menopausal status } & \\ \text { Premenopausal } & 97(75.78) \\ \text { Postmenopausal } & 31(24.22) \\ \text { Primary site } & \\ \text { Stomach } & 41(32.03) \\ \text { Colon and rectum } & 58(45.31) \\ \text { Breast } & 8(6.25) \\ \text { Small intestine } & 5(3.92) \\ \text { Gallbladder } & 4(3.12) \\ \text { Vermiform appendix } & 4(3.12) \\ \text { Unknown } & 8(6.25) \\ \text { Ovarian involvement } & \\ \text { Bilateral } & 98(76.56) \\ \text { Unilateral } & 30(23.44) \\ \text { Tumor diameter, cm } & \\ \text { Median (range) } & 9.6(4-18) \\ \leq 5 & 11(8.59) \\ 5-10 & 42(32.81) \\ \geq 10 & 75(58.60) \\ \end{array}$

Chronology

Synchronous

$36(28.13)$

Metachronous

$92(71.87)$

Extent of disease

Ovary

$71(55.47)$

Pelvis

34 (26.56)

Beyond pelvis

$23(17.97)$

Chemotherapy

Yes

$89(69.53)$

No

$39(30.47)$

Ascites

Yes

$63(49.22)$

No

$65(50.78)$

Metastasectomy

Yes

$114(89.06)$

No

14 (10.94)

was longer compared with that for tumors of gastric origin (31 vs. 11 months, respectively; $\mathrm{P}<0.0001$ ). Among all patients, those with metachronous cancer exhibited a longer mean survival time compared with those who exhibited synchronous metastases $(\mathrm{P}=0.0113)$. The patients with metastatic disease confined to the ovaries had a median survival time of 23 months compared with 13.5 months for those with more extensive metastasis $(\mathrm{P}<0.0001)$ (Table II). By contrast, no correlation was observed between patient age and survival. Menopausal status, bilaterality and size of ovarian metastases were also included in the univariate analysis; however, these 
Table II. Prognostic factors for Krukenberg tumor.

\begin{tabular}{|c|c|c|c|c|}
\hline \multirow[b]{2}{*}{ Factors } & \multicolumn{2}{|c|}{ Univariate analysis } & \multicolumn{2}{|c|}{ Multivariate analysis } \\
\hline & $\operatorname{HR}(95 \% \mathrm{CI})$ & P-value & $\operatorname{HR}(95 \% \mathrm{CI})$ & P-value \\
\hline Age ( $\leq 50$ vs. $>50$ years $)$ & $1.121(0.607-1.310)$ & 0.5599 & - & - \\
\hline Menopausal status (pre- vs. postmenopausal) & $0.844(0.786-1.787)$ & 0.4171 & - & - \\
\hline Primary site (stomach vs. colon and rectum) & $0.124(0.074-0.208)$ & $<0.0001$ & $0.252(0.135-0.469)$ & $<0.0001$ \\
\hline Primary site (stomach vs. breast) & $0.059(0.025-0.141)$ & $<0.0001$ & $0.125(0.046-0.339)$ & $<0.0001$ \\
\hline Primary site (stomach vs. others) & $0.249(0.131-0.474)$ & $<0.0001$ & $0.389(0.182-0.833)$ & 0.0151 \\
\hline Ovarian involvement (bilateral vs. unilateral) & $1.316(0.503-1.148)$ & 0.1916 & - & - \\
\hline Tumor diameter ( $\geq 10$ vs. $<10 \mathrm{~cm})$ & $0.905(.0464-1.767)$ & 0.7708 & - & - \\
\hline Chronology (metachronous vs. synchronous) & $1.701(1.127-2.564)$ & 0.0113 & $1.898(1.182-3.049)$ & 0.0080 \\
\hline Extent of disease (ovary vs. pelvis) & $5.486(3.272-9.198)$ & $<0.0001$ & $2.156(1.170-3.974)$ & 0.0138 \\
\hline Extent of disease (ovary vs. beyond pelvis) & $12.702(6.965-23.166)$ & $<0.0001$ & $0.856(0.307-2.387)$ & 0.7666 \\
\hline Chemotherapy (no vs. yes) & $0.293(0.195-0.440)$ & $<0.0001$ & $0.626(0.371-1.057)$ & 0.0796 \\
\hline Ascites (no vs. yes) & $7.816(4.913-12.436)$ & $<0.0001$ & $4.820(2.537-9.157)$ & $<0.0001$ \\
\hline Metastasectomy (yes vs. no) & $9.346(4.950-17.544)$ & $<0.0001$ & $4.878(1.572-15.15)$ & 0.0060 \\
\hline
\end{tabular}

HR, hazard ratio; CI, confidence interval.

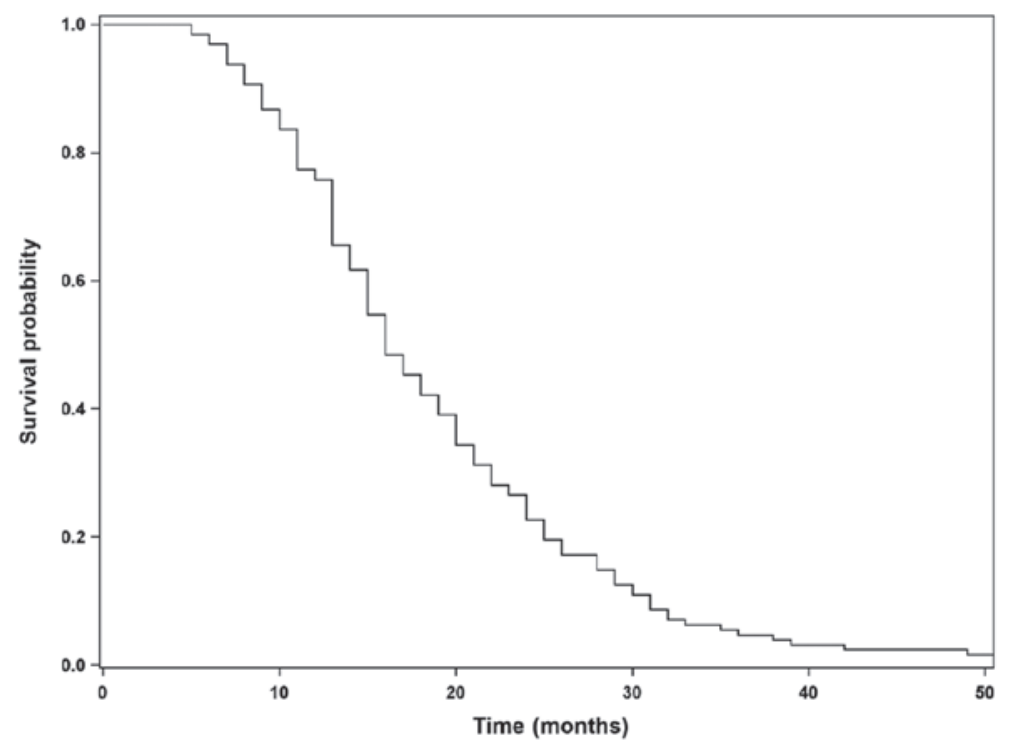

Figure 1. Overall survival (OS) curve for all 128 patients. The median OS was 16 months (range, 5-52 months).

factors were not identified as significant prognostic indicators for OS (P>0.05) (Table II).

The multivariate Cox regression analysis demonstrated that synchronous metastasis (HR=1.898, 95\% CI: 1.182-3.049, $\mathrm{P}=0.008)$, pelvic invasion $(\mathrm{HR}=2.156,95 \% \mathrm{CI}: 1.170-3.974$, $\mathrm{P}=0.0138)$, ascites $(\mathrm{HR}=4.820,95 \% \mathrm{CI}: 2.537-9.157, \mathrm{P}<0.0001)$ and no metastasectomy $(\mathrm{HR}=4.878,95 \% \mathrm{CI}: 1.572-15.15$, $\mathrm{P}=0.0060)$ were independent factors for predicting an unfavorable OS (Table II and Fig. 2).

\section{Discussion}

The outcome and prognosis of the 128 patients with Krukenberg tumor included in our study reflects the complexity and challenges in the management of this patient population. The prognosis of Krukenberg tumor is dismal and the benefit of ovarian metastasectomy remains to be elucidated.

Although Krukenberg tumors may be induced by complex mechanisms, lymph node metastasis is considered to be the most significant risk factor for recurrence. It was reported that patients with Krukenberg tumor were younger compared with those who had primary ovarian cancer, whereas the functioning ovary was prone to metastatic disease due to the rich ovarian blood supply predisposing to hematogenous metastasis (6). Several mechanisms have been suggested to explain the progression and recurrence pathway of gastric cancer, such as lymphatic spread, hematogenous spread, direct invasion and peritoneal seeding. Among these, the incidence of hematogenous recurrence is the 
A

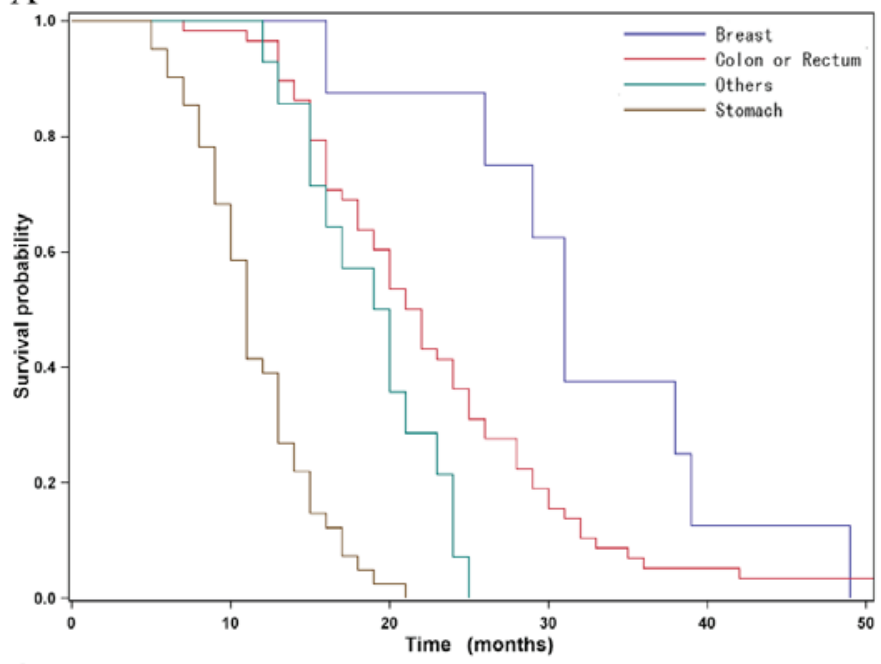

$\mathrm{C}$

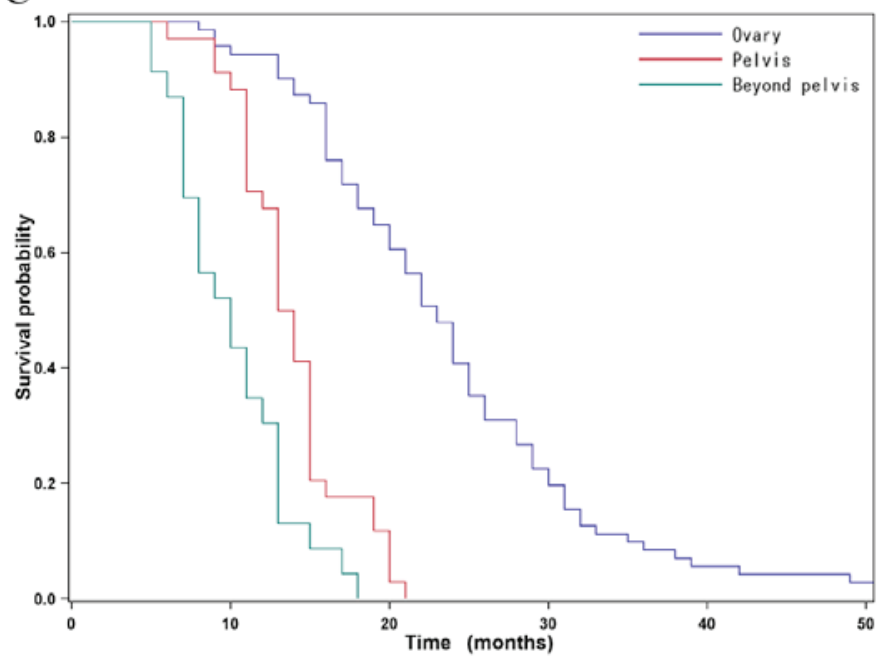

$\mathrm{E}$

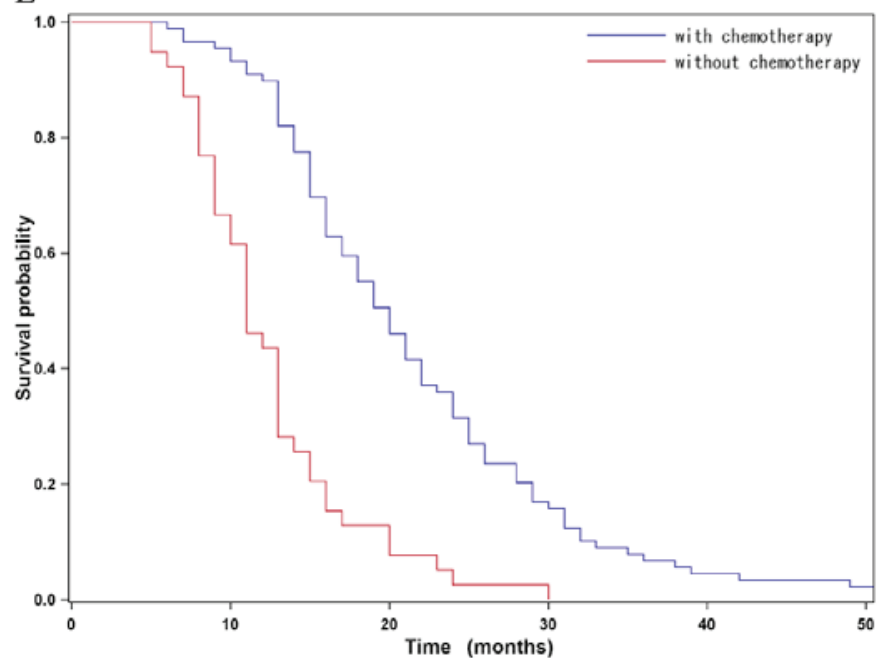

B

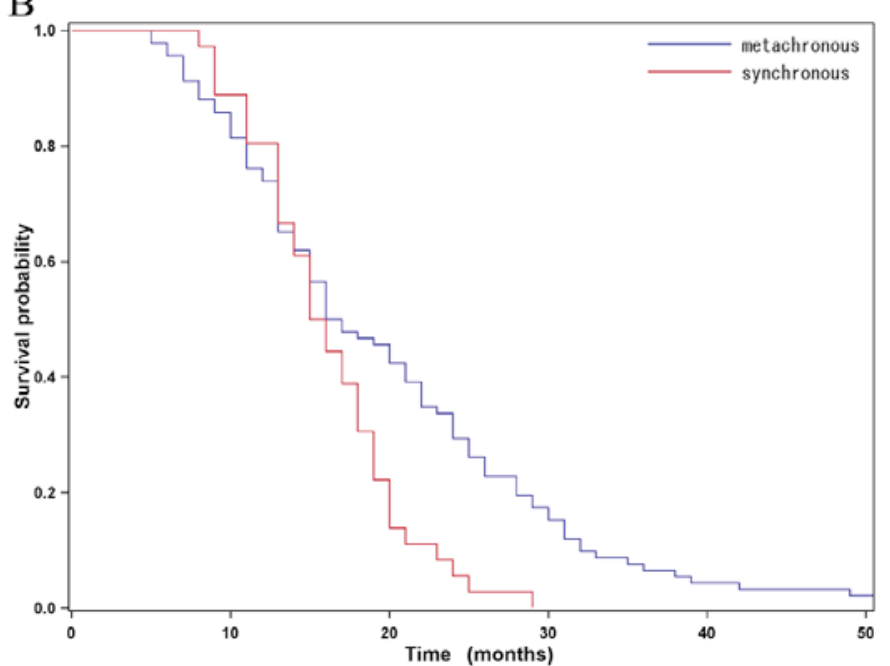

$\mathrm{D}$

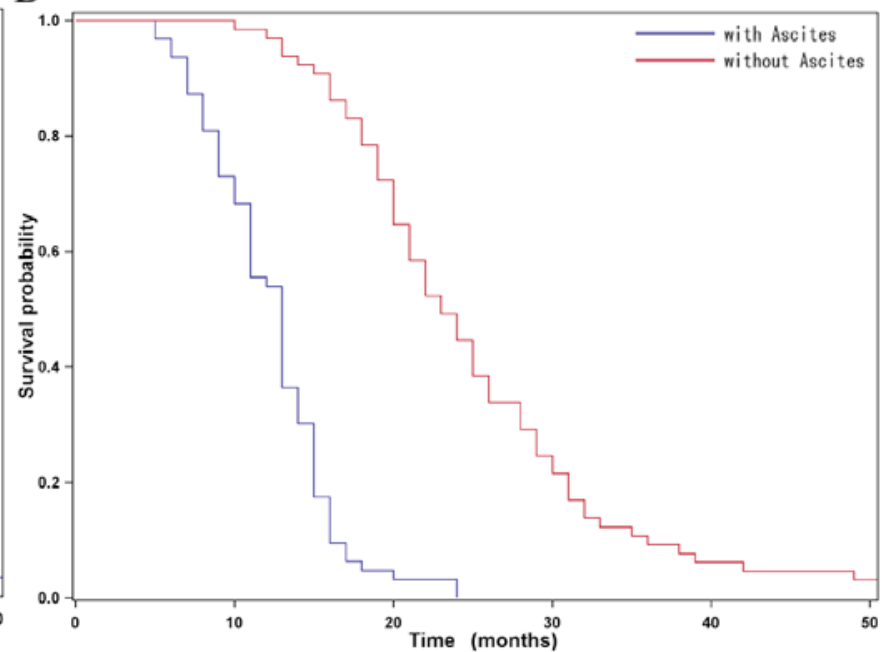

$\mathrm{F}$

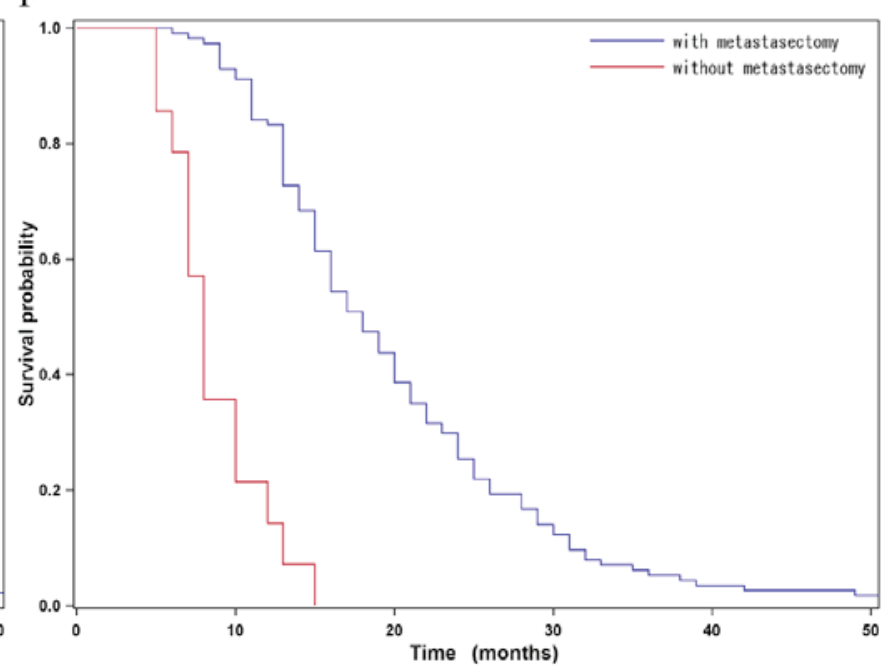

Figure 2. Survival curves of patient subgroups according to prognostic factors. (A) Patients with different primary sites (P<0.0001). (B) Patients with synchronous or metachronous disease $(\mathrm{P}=0.008)$. (C) Patients with or without disease beyond the ovary $(\mathrm{P}=0.0138)$. ( $\mathrm{D}) \mathrm{Patients}$ with or without ascites $(\mathrm{P}<0.0001)$. (E) Patients with or without chemotherapy $(\mathrm{P}=0.0796)$. (F) Patients with or without metastasectomy $(\mathrm{P}=0.0060)$.

highest (7,8). The exact mechanism of the spread of breast cancer to the ovaries had not been elucidated, but the risk of primary ovarian cancer is increased in women with breast-ovarian cancer syndrome, which is caused by BRCA1/2 mutations (9).
The most common primary tumor site in patients with Krukenberg tumor is reportedly the stomach $(1,10,11)$. However, recent observations have reported a higher incidence of colorectal rather than gastric origin and, in particular, more 
frequently from the colon rather than the rectum (12), which was consistent with the findings in our study, as over half of our patients exhibited primary colorectal cancer. Radiotherapy for rectal cancer with lymphovascular invasion may contribute to reducing the risk of ovarian spread.

The survival of patients with Krukenberg tumor is associated with the primary tumor site. In our study, patients with tumors originating in the breast exhibited the longest median OS of 31 months, followed by those with cancer of colorectum, with a median survival time of 21.5 months; the prognosis of patients with a gastric origin was the poorest (median OS, 11 months), which was comparable with previous data (13). The possible explanations are as follows: i) The prognosis of advanced gastric cancer is worse compared with that of advanced colorectal cancer; and ii) patients with Krukenberg tumor of gastric origin usually exhibit a lower performance status score and severe anemia. Furthermore, breast cancer is generally associated with a better prognosis compared with tumors of the gastrointestinal tract.

In the present study, we identified synchronous ovarian metastasis as an independent risk factor associated with poor survival. It was previously reported that synchronous ovarian metastasis was an unfavorable factor correlated with poor survival (14), which was consistent with our study, suggesting that the metastasis-free interval was shortened between primary tumor diagnosis and ovarian metastasis.

The survival of patients without ascites was significantly longer compared with that of patients with ascites (median OS, 23 vs. 13 months, respectively; $\mathrm{P}<0.0001$ ) and ascites was found to be an independent risk factor associated with poor survival. These results were consistent with those of other studies $(15,16)$. In Krukenberg tumor patients, ascites may be caused by tumor invasion of the peritoneum or malnutrition, and it is usually associated with dissemination to the abdominal or pelvic cavity. Peritoneal dissemination was reported as an adverse factor affecting survival $(17,18)$. In our analysis, high incidence of pelvic invasion or extension beyond the pelvis were also determined as poor prognostic factors. Additionally, the fact that there was no survival difference according to tumor size or bilaterality indicates that the development of ovarian metastases is a sign of more aggressive disease and ovarian metastases are diagnosed late during cancer progression.

Several studies have investigated the options of metastasectomy and cytoreductive surgery for Krukenberg tumor $(13,19,20)$. The role of metastasectomy for Krukenberg tumor was assessed between different primary cancer types, different types of surgery or extent of residual disease. Bilateral oophorectomy for Krukenberg tumor have been shown to positively affect OS in isolated ovarian metastasis patients in an Italian study (21). Cheong et al (22) reported on 54 patients with Krukenberg tumors who experienced disease relapse following curative surgery of primary gastric cancer. Of the 54 patients who underwent resection of the Krukenberg tumor, the 33 who underwent metastasectomy exhibited a significantly longer median OS compared with those who did not undergo metastasectomy (17 vs. 3 months, respectively). In our study, metastasectomy was also a beneficial prognostic factor in terms of OS. Therefore, resection of metastatic ovarian tumors and cytoreductive surgery as part of the treatment for Krukenberg tumor play a pivotal role in prolonging the survival time of the patients, provided that there is no distant metastasis. In addition to surgical treatment, chemotherapy is also an option. Palliative radiotherapy may be applied for unresectable or distant metastatic Krukenberg tumors. In order to improve survival, there is a need to investigate the optimal management of Krukenberg tumors. For patients with gastric cancer, a Korean study suggested that debulking or gastrectomy plus metastasectomy may achieve survival benefits for patients with distant metastases who were receiving systemic chemotherapy (23). In our study, over two-thirds of the patients received chemotherapy. The majority of the chemotherapeutic regimens included a platinum agent (cisplatin, carboplatin or oxaliplatin) plus 5-fluorouracil, whereas other patients received docetaxel and paclitaxel, with distinct survival benefits.

In conclusion, in our study, Krukenberg tumors more commonly appeared to originate from primary gastrointestinal tract tumors. The prognosis of Krukenberg tumor is dismal and patients may benefit from ovarian metastasectomy. Metastasis outside the ovaries, ascites and no metastasectomy were independent factors for predicting an unfavorable OS. As Krukenberg tumors are rather rare, a national registry should be created to collect information on these patients, with the aim to improve diagnosis and treatment outcome. The identification of the primary tumor is crucial for designing an effective treatment regimen for this group of patients, whereas imaging examinations and gastrointestinal endoscopy are recommended prior to ovarian metastasectomy.

\section{Acknowledgements}

The authors would like to thank the Department of Oncology, the First Affiliated Hospital of Xi'an Jiaotong University School of Medicine, Tumor Hospital of Shaanxi and the Department of Oncology, People's Hospital of Shaanxi, for their assistance with stastistical analysis. The authors also acknowledge the efforts of the dedicated study volunteers.

\section{References}

1. Al-Agha OM and Nicastri AD: An in-depth look at Krukenberg tumor: An overview. Arch Pathol Lab Med 130: 1725-1730, 2006.

2. Young RH: From Krukenberg to today: The ever present problems posed by metastatic tumors in the ovary: Part I. Historical perspective, general principles, mucinous tumors including the Krukenberg tumor. Adv Anat Pathol 13: 205-227, 2006.

3. Novak C and Gray LA: Krukenberg tumor of the ovary: Clinical and pathological study of four cases. Surg Gynecol Obstet 66: 157-165, 1938.

4. Serov SF and Scully RE (eds): Histological typing of ovarian tumors. In: International Histological Classification of Tumours. Vol. 9. WHO, Geneva, 1973.

5. Lu W, Yuan L, Liu X and Guo SW: Identification of prognostic factors for Krukenberg tumor. GMIT 2: 52-56, 2013.

6. La Fianza A, Alberici E, Pistorio A and Generoso P: Differential diagnosis of Krukenberg tumors using multivariate analysis. Tumori 88: 284-287, 2002.

7. Yoo CH, Noh SH, Shin DW, Choi SH and Min JS: Recurrence following curative resection for gastric carcinoma. Br J Surg 87: 236-242, 2000.

8. Shin DW, Hyung WJ, Noh SH and Min JS: Risk factors for recurrence after curative surgery for early gastric cancer. J Korean Gastric Cancer Assoc 1: 106-112, 2001.

9. Kauff ND and Barakat RR: Risk-reducing salpingo-oophorectomy in patients with germline mutations in BRCA1 or BRCA2. J Clin Oncol 25: 2921-2927, 2007. 
10. Januszewska M, Emerich J, Dibniak J, Sliwinski W and Stukan M: Clinical analysis of patients with Krukenberg tumor of the ovary. Ginekol Pol 77: 203-208, 2006 (In Polish).

11. Kiyokawa T, Young RH and Scully RE: Krukenberg tumors of the ovary: A clinicopathologic analysis of 120 cases with emphasis on their variable pathologic manifestations. Am J Surg Pathol 30: 277-299, 2006.

12. Moore RG, Chung M, Granai CO, Gajewski W and Steinhoff MM: Incidence of metastasis to the ovaries from nongenital tract primary tumors. Gynecol Oncol 93: 87-91, 2004.

13. Jiang R, Tang J, Cheng X and Zang RY: Surgical treatment for patients with different origins of Krukenberg tumors: Outcomes and prognostic factors. Eur J Surg Oncol 35: 92-97, 2009.

14. Xue Z, Yan H, Li J, Liang S, Cai X, Chen X, Wu Q, Gao L, $\mathrm{Wu} \mathrm{K}$, Nie Y, et al: Identification of cancer stem cells in vincristine preconditioned SGC7901 gastric cancer cell line. J Cell Biochem 113: 302-312, 2012.

15. Li W, Wang H, Wang J, L v F, Zhu X and Wang Z: Ovarian metastases resection from extragenital primary sites: Outcome and prognostic factor analysis of 147 patients. BMC Cancer 12 : 278, 2012.

16. Peng W, Hua RX, Jiang R, Ren C, Jia YN, Li J and Guo WJ: Surgical treatment for patients with Krukenberg tumor of stomach origin: Clinical outcome and prognostic factors analysis. PLoS One 8: e68227, 2013

17. Kim HK, Heo DS, Bang YJ and Kim NK: Prognostic factors of Krukenberg's tumor. Gynecol Oncol 82: 105-109, 2001.
18. Yonemura Y, Bandou E, Kinoshita K, Kawamura T, Takahashi S, Endou Y and Sasaki T: Effective therapy for peritoneal dissemination in gastric cancer. Surg Oncol Clin N Am 12: 635-648, 2003

19. Ayhan A, Guvenal T, Salman MC, Ozyuncu O, Sakinci M and Basaran M: The role of cytoreductive surgery in nongenital cancers metastatic to the ovaries. Gynecol Oncol 98: 235-241, 2005.

20. Kim WY, Kim TJ, Kim SE, Lee JW, Lee JH, Kim BG and Bae DS: The role of cytoreductive surgery for non-genital tract metastatic tumors to the ovaries. Eur J Obstet Gynecol Reprod Biol 149: 97-101, 2010.

21. Erroi F, Scarpa M, Angriman I, Cecchetto A, Pasetto L, Mollica E, Bettiol M, Ruffolo C, Polese L, Cillo U, et al: Ovarian metastasis from colorectal cancer: Prognostic value of radical oophorectomy. J Surg Oncol 96: 113-117, 2007.

22. Cheong JH, Hyung WJ, Chen J, Kim J, Choi SH and Noh SH: Survival benefit of metastasectomy for Krukenberg tumors from gastric cancer. Gynecol Oncol 94: 477-482, 2004.

23. Kim KH, Lee KW, Baek SK, Chang HJ, Kim YJ, Park J, Kim JH, Kim HH and Lee JS: Survival benefit of gastrectomy \pm metastasectomy in patients with metastatic gastric cancer receiving chemotherapy. Gastric Cancer 14: 130-138, 2011. 